\title{
Optical Modulator P/N Junction Mapping by Electron Holography and Scanning Capacitance Microscopy
}

\author{
Y.Y. Wang, J. Nxumalo, J. Jeon, K. Barton, and K. Nummy \\ GlobalFoundires Inc., Hopewell Junction, NY, USA
}

The electron holography and scanning capacitance microscopy have successfully been used to map the junction profile for semiconductor device [1-4] over the years. A schematic of electron holography setup is shown in fig.1a, where electron beam passes through the sample interfere with the reference beam and form an interference fringe pattern over the image of the sample. In fig. $1 \mathrm{~b}$, it shows a phase shift of the fringe when the holography fringe overlays on top of different type of semiconductor material. The electric potential is proportional to the phase shift, which is also related to the dopant concentration as shown in fig. 2 for Si at room temperature based on theoretical calculation [5].

Scanning capacitance microscopy $(\mathrm{SCM})$ is a $2 \mathrm{D} \mathrm{P} / \mathrm{N}$ junction profiling technique used for mapping active dopant on semiconductor surfaces. It utilizes a modified atomic force microscope (AFM) by employing a conductive probe tip attached to a capacitance sensor (Fig 3a). Fig. 3b illustrates that SCM measures local depletion capacitance vs voltage $(d C / d V)$ where the phase of the signal represents the type of carriers and the magnitude represents the concentration of carriers. Fig. 4 shows active carrier concentration in relationship with $d C / d V$ value. The positive sign of $d C / d V$ indicates p-type carrier and negative sign indicates n-type carrier. The SCM probe tip is raster scanned across the sample surface and allows spatially resolved images of carrier types and concentration with high spatial resolution $(\sim 5 \mathrm{~nm})$.

Optical modulator is a critical part of Si photonics circuit, where electric signal is converted into optical signal [6]. One of the modulator design is made of light doped p-n junctions which connects with high doped $\mathrm{N}+$ and $\mathrm{P}+$ on the left and right side for the contact, shown in fig.5a. Since this device has $\mathrm{N}+, \mathrm{N}-, \mathrm{P}-$ and $\mathrm{P}+$ dopant at close vicinity, it is an ideal device to study how electric potential and carrier density changes with the dopant concentration by electron holography and SCM. Fig.5b is an electric potential map (phase map) measured by electron holography: right side is the $\mathrm{P}+$ dopant contact with $-0.5 \mathrm{rad}$ phase shift and left side is the $\mathrm{N}+$ dopant contact with $+0.5 \mathrm{rad}$ phase shift. The middle section is the optical modulator $\mathrm{P} / \mathrm{N}$ junction with a smaller phase shift difference between $\mathrm{N}$ - and $\mathrm{P}$-. The phase shift steps down from left to right with $\mathrm{N}+, \mathrm{N}-, \mathrm{P}-$ and $\mathrm{P}+$ as dopant concentration changes, which is consistent with the plot of the electric potential vs. dopant concentration shown in Fig.2. Fig.5c is an SCM measurement, shown a P-N junction in the middle section for the optical modulator with $\mathrm{N}-/ \mathrm{P}$ - dopant. On the right, a smaller positive $d C / d V$ value indicates $\mathrm{P}+$ dopant and on the left, a small negative $d C / d V$ value indicates $\mathrm{N}+$ dopant. Overall, these two techniques provide a consistent picture for optical modulator diode with a minor difference at $\mathrm{P}$ - region in the middle section. Electron holography data show an inverted layer at bottom of Si, while SCM data do not show that inversion layer. The inversion layer is due to the positive charge at bottom of crystalline $\mathrm{Si}\left(\mathrm{Si} / \mathrm{SiO}_{2}\right.$ interface). The electron holography with $400 \mathrm{~nm}$ thick sample is a bulk technique, while the SCM is a surface technique, which may not be sensitive to the charge at bottom of Si [7].

\section{Reference:}

[1] W.D. Rau et al, Phys. Rev. Lett. 82 (1999), p. 2614.

[2] Y.Y. Wang et al, Ultramicroscopy 101 (2004), p. 63.

[3] Y.Y. Wang et al, Ultramicroscopy 124 (2013), p. 117.

[4] C.C. Williams et al, J. Vac. Sci. Technol. A 8 (1990), p. 895. 
[5] M. Sze, Physics of Semiconductor Devices, Second Edition

[6] F. Gardes et al, Proc. of SPIE, 7608 (2010), p.76080J-1

[7] We would like to thank GlobalFoundries managers, E. Crawford and A. Katnani, for their support of this work.
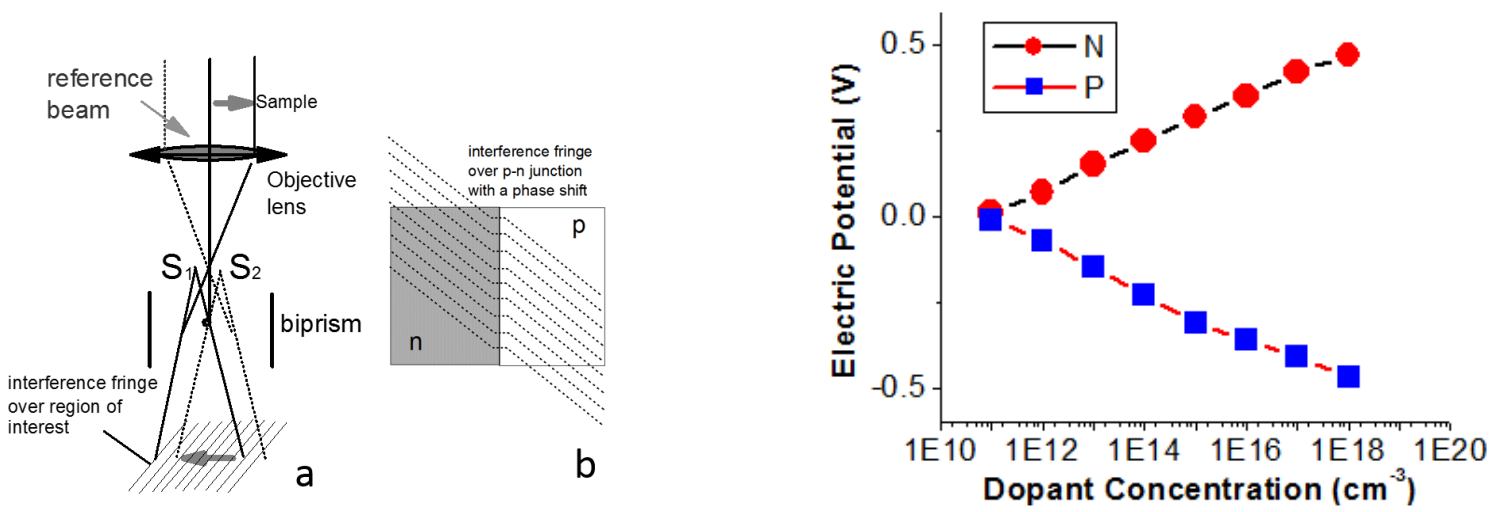

Figure 1. Electron holography set up. Figure 2. Electric potential vs. dopant concentration at $\mathrm{T}=300^{\circ} \mathrm{K}$

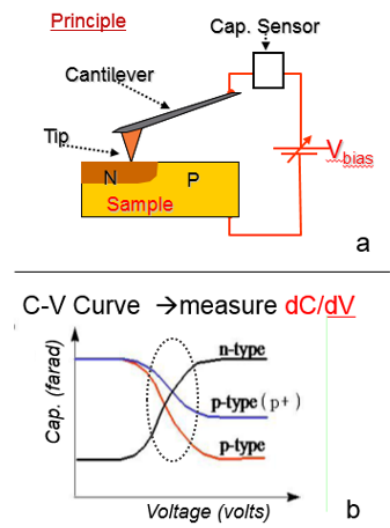

Figure 3. SCM setup

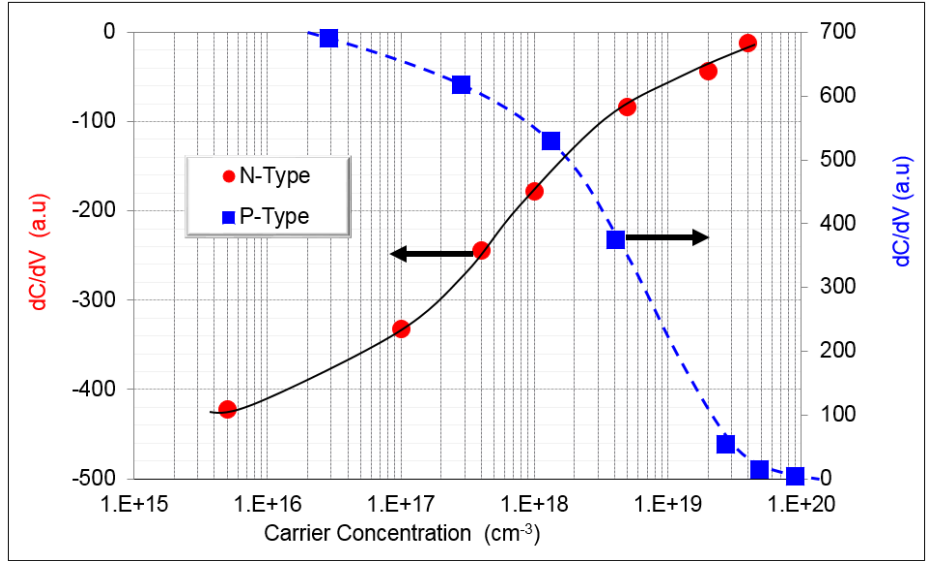

Figure 4. $d C / d V$ value vs. active dopant concentration for SCM.
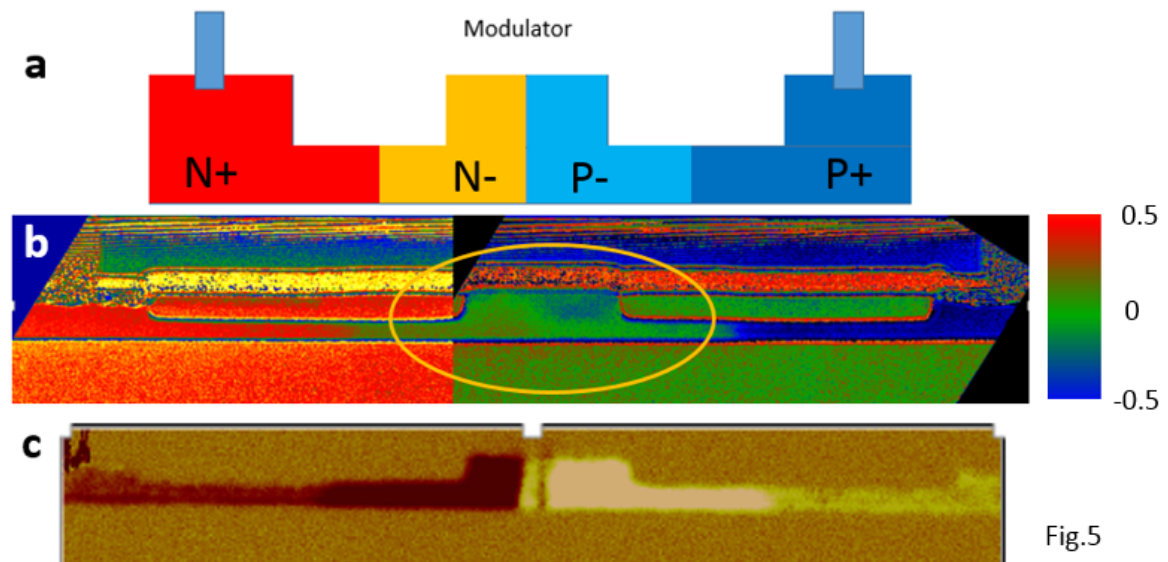

Fig.5

Figure 5. a). Schematics of an optical modulator; b). Phase map by electron holography; c). $d C / d V$ map by SCM. 